\title{
Clinical Applications and Properties of Calcium Citrate Malate
}

Bharat Kwatra*, Angarika Balakrishnan ${ }^{1}$, Naushin Raheema ${ }^{2}$, Navnoor Kaur ${ }^{3}$, Nilasha Chakrabarty ${ }^{4}$, Shakila Mumtaz $^{5}$

${ }^{*}$ Corresponding Author, Invenzion Labs Inc, New Delhi, India.

${ }^{1}$ Department of Biological Sciences, Sunandan Divatia School of Science, SVKM's NMIMS (Deemed-to-be University), Vile Parle (west), Mumbai 400056, India.

2 Lumina Datamatics Limited, Chennai, Tamilnadu, India.

${ }^{3}$ University of Delhi, Delhi, India.

${ }^{4}$ St. Xaviers College, Kolkata, West Bengal, India.

5 The limit group of college, Sahiwal, India.

*Corresponding author's E-mail: bkwatra999@gmail.com

Received: 11-04-2021; Revised: 25-06-2021; Accepted: 30-06-2021; Published on: 15-07-2021.

\section{ABSTRACT}

This article is an examination of the Clinical applications and properties of Calcium Citrate Malate. The scientific development and subsequent need to understand the properties of Calcium Citrate Malate, that make it an excellent candidate for treatment of disorders in various clinical domains, continues to influence the researchers all over the globe today. This article examines the research done and published by researchers and scientists. Consideration of current trends and data in scientific queries and demonstrates further aspects of the clinical applications and properties of Calcium Citrate Malate. Additionally, this article explores options for the role of Calcium Citrate Malate supplementation in dental care, to prevent tooth loss, erosion and abrasion, in Immunology as a critical signal for inflammation, in joints to treat osteoarthritis and in nephrology to tackle the renal stone problem.

Keywords: CCM, Nephrology, Joints, Dental, Immunology.

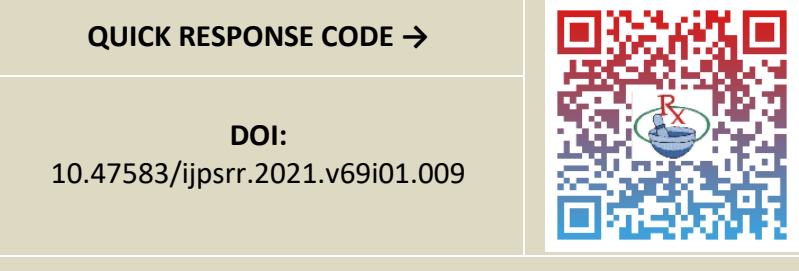

DOI link: http://dx.doi.org/10.47583/ijpsrr.2021.v69i01.009

\section{INTRODUCTION}

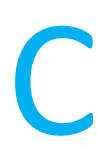

alcium is an important mineral which is required by humans to maintain strong bones and teeth, move muscles and for nerve conduction. Sources of calcium include milk, green leafy vegetables, fish and anything made with fortified flour. Hypocalcaemia may result due to a number of reasons, this calcium deficiency can lead to weak nails, slower hair growth, fragile skin and in worst cases seizures.

In order to tackle this ever-growing problem of calcium deficiency, researchers devised a supplement in the form of calcium citrate Malate. This complex is water soluble and when it dissolves in water it releases calcium ions and a calcium citrate complex. The calcium ions gets absorbed via the intestinal mucosa whereas the citrate is introduced into the body via paracellular absorption. The questions arises as to why is Calcium citrate Malate preferred over calcium citrate? This is because the structure of calcium citrate Malate increases its dissolution in the stomach by 6-9 times as compared to calcium citrate, additionally it is well absorbed even when taken without food. It has also shown to have superior bioavailability than other calcium compounds.

Calcium Citrate Malate has been shown to have several applications such as treating Osteoporosis, Osteomalacia, hypoparathyroidism and certain muscle disorders such as latent tetany. It helps to maintain bone mass and significantly decreases the risk of bone fracture in the elderly. It is also recognized as a source of calcium that does not increase the risk of kidney stone formation; in fact, it protects the consumer against stone formation.

With the change in the lifestyle of people, owing to the pandemic associated lockdown, people are resorting the supplements to maintain a healthy lifestyle, boost immunity to offer some protection against the novel Coronavirus. Thus, this review summarizes a few of the health benefits associated with Calcium Citrate Malate such as its positive effects on dental care, Immunity, nephrology and joints.

\section{METHODOLOGY}

The study was conducted using four databases Google Scholars SAGE, DOAJ and PubMed. Selection of papers were done based on keywords and theme relevant to this review. Further the published papers from these databases were arranged in systematic order with respect to the year of publication. 


\section{RESULTS AND DISCUSSION}

\subsection{Calcium Citrate Malate in Dental Care ${ }^{1-9}$}

\begin{tabular}{|c|c|c|}
\hline $\begin{array}{l}\text { Sr. } \\
\text { No. }\end{array}$ & Title of paper & $\begin{array}{l}\text { Year } \\
\text { Publis } \\
\text { hed }\end{array}$ \\
\hline 1 & $\begin{array}{l}\text { Increased risk of tooth loss is related to bone } \\
\text { loss at the whole body, hip, and spine }\end{array}$ & 1996 \\
\hline 2 & Lessening dental erosive & 1996 \\
\hline 3 & $\begin{array}{l}\text { Comparison of Erosion of Dental Enamel by } \\
\text { Four Drinks Using an Intra-Oral Appliance }\end{array}$ & 1996 \\
\hline 4 & $\begin{array}{l}\text { Calcium and vitamin D supplements reduce } \\
\text { tooth loss in the elderly }\end{array}$ & 2001 \\
\hline 5 & Tooth wear: Attrition, erosion, and abrasion. & 2003 \\
\hline 6 & $\begin{array}{l}\text { The Health benefits of Calcium citrate Malate: } \\
\text { A Review of the Supporting Science }\end{array}$ & 2008 \\
\hline 7 & $\begin{array}{l}\text { Effect on oral } \mathrm{pH} \text { changes and taste perception } \\
\text { in } 10-14 \text {-year-old children, after calcium } \\
\text { fortification of a fruit juice }\end{array}$ & 2015 \\
\hline 8 & Calcium and Calcium salts & 2017 \\
\hline 9 & Macro elements: $\mathrm{Ca}, \mathrm{Na}, \mathrm{K}, \mathrm{P}, \mathrm{Cl}$. & 2020 \\
\hline
\end{tabular}

An increased risk of tooth loss is linked to bone loss throughout the body, including the hip and spine.

Increased systemic bone loss, which contributes to the resorption of tooth-supporting alveolar bone, maybe a risk factor for tooth loss. Simultaneous longitudinal connections between tooth loss and bone loss at the whole body, femoral neck, and spine were investigated in 189 healthy, white, postmenopausal women who took part in three intervention trials over 7 years. No one in the study was taking oestrogen. Dual photon or dual-energy X-ray absorptiometry was used to determine the bone mineral density (BMD). Teeth were counted at baseline, and the number and timing of teeth lost during the evaluation period were analysed using a list of questions. All analyses were subjected to year-by-year control. This study extends the cross-sectional findings by describing the correlations between tooth loss and bone loss at the whole body, hip, and spine in 189 of those women who were observed for 7 years after they participated in the original study. The study's goals were to see if levels of bone loss in the entire body, femoral neck, and spine are increased in women who are also losing teeth and to predict the impact of tooth loss associated with systemic bone loss. In summary, higher rates of BMD loss at different skeletal sites were linked to tooth loss in healthy postmenopausal women. These establishments were unaffected by the number of years since menopause, BMI, number of teeth at baseline, smoking habits, or calcium and vitamin $D$ regimes. The risk of tooth loss increased with the number of teeth at baseline and the number of years since menopause. In this longitudinal study, the temporal relationship between tooth loss and bone loss at the whole body, hip, and spine tested the assumption that systemic bone loss may play a fundamental role in tooth loss in postmenopausal women ${ }^{1}$.
Modification of Product by reducing the potentiality of dental erosion.

Due to the obvious increasing importance in dental erosion, there is a greater focus on how highly erosive materials can be altered. Knowledge on how to implement this has been difficult to come by, and has primarily focused on the possibility of redesigning soft drinks. The majority of the published work on this topic relates to calcium and phosphate supplementation, ranging from early experiments on saturation of a demineralizing medium with tricalcium phosphate, to tests of more soluble phosphates and other calcium salts providing various levels of $\mathrm{Ca} 2+$ and PO4", to a calcium citrate Malate additive particularly developed. Interpretations on the efficiency of citrate, the feasibility of lowering the acidity levels of soft drinks, and the potential applications of fluoride, bicarbonates, and certain components of milk products are also included ${ }^{2}$.

\section{Usage of Intra-Oral Appliance for Comparing the Erosion of Dental Enamel by Four Drinks.}

Dental erosion is caused by the increased consumption of acidic foods and soft drinks. In this study the ability of an intra-oral enamel slab system is done and the citric acidbased orange drink is compared and measured for erosive potential with and without adding the CCM. Eleven adults wore the intra-oral appliance four times per day for 6 days. Each of the appliance held two enamel slabs which were subjected to the test drinks for 15 mins four times a day for 6 days. The loss of enamel were quantified by profiling the casts of enamel slabs taken before and after the subjection to the test. The loss of enamel was great during the exposure to a acid-based cola drink. The exposure was same during the test of citric acid-based orange drink with and without CCM. The results indicated that it was not possible to determine if $\mathrm{CCM}$ had the potential to reduce the erosion as there was not much difference in statistical significance. Although the experimental set-up could have been changed for better outcomes. The enamel slab system was able to differentiate between drinks according to their erosive potential. The effect of CCM as an antierosion agent was not able to interpret in this study due to unavailability of erosive potential of the citric acid-based orange drink ${ }^{3}$.

\section{Prevention of tooth loss in elder people with supplementation of calcium and Vitamin D.}

Calcium and Vitamin D supplementation decreases the rate of bone loss from different skeletal sites. In the current research, individuals aged more than 65 were examined and were already placed under and completed a 3 year randomized placebo controlled trial of the effect of both Calcium and Vitamin D3. During this trial, 11 individuals taking supplements and 17 individuals intaking placebo lost one or more teeth. In conclusion, calcium and Vitamin D supplements were implicated as a cause of tooth loss in elder men and women. These findings must be confirmed in a clinical trial where the tooth and oral bone 
loss are the key targets and extra risk factors for tooth loss are quantified throughout the trial. If nutritional supplementation is proven to be effective, these low-cost and easily accessible measures could have a significant impact on the public's oral health ${ }^{4}$.

\section{The types of tooth wear: Attrition, Abrasion and Erosion}

There are three different aetiologies for tooth wear; however, several studies suggest a multifactorial aetiology, which obscures the delineation of each aetiology. These causes may act sequentially or consecutively, therapeutically or additively, or in parallel with other entities to conceal the true nature of tooth wear. Treatment may be required in some cases to halt the progression of tooth wear. Non-carious mechanisms can provoke tooth structure loss. Individuals' regressive adjustments may differ in aetiology, extent, and treatment response, and they may be correlated with physiologic or pathologic procedures. Those entities have generally been categorised as attrition, erosion, and abrasion. Attrition is the metabolic loss of tooth structure as a result of masticatory methods; erosion is the synthetic disintegration of the framework that does not require the carious process, and abrasion is the mechanical wearing away of structure ${ }^{5}$.

\section{A review on health benefits of Calcium Citrate Malate}

Calcium citrate Malate has been under research for many years and it was patented in the late 1980s. CCM has many roles such as it lessens the risk of bone fracture in the elderly people, contains nutritional benefits for people of all ages. CCM is defined as a compound salt of Ca cation with a combination of citrate and Malate anion. Other $\mathrm{Ca}$ salts do not exhibit the qualities of CCM like high aqueous solubility, enhanced absorbability, and bioavailability. This review provides a good perspective on CCM being better than $\mathrm{Ca}$ in most of the views. Calcium citrate Malate is increasingly bioavailable as a calcium source when added to food and other dietary supplements when compared to other Ca salts. CCM is used as a good fortification component for food and beverages. It is best known fortification agent for fruit juice, OJ, milk, plant milks, fitness waters, juice blends, etc. CCM has been proved to be highly absorbable as both tablet and beverage form. Though CCM is a better fortificant, it does not provide the desirable taste in cola-flavoured drinks. CCM has exceptional benefits. It has shown great effect in minimizing the tooth erosion and inducing the tooth retention. CCM has other properties such as reduction of blood pressure, colon health, and it does not increase the risk of kidney stone and it protects from forming the stones. Though there are various benefits, it is said that CCM is high in cost when compared to other Ca resources ${ }^{6}$.

\section{Impacts of calcium fortification of a fruit juice on oral $\mathrm{pH}$ alterations and taste interpretation in 10-14-year-old children}

The purpose of this study was to see how calcium-fortified juices influenced oral $\mathrm{pH}$ changes and taste perception in children aged 10-14. The null hypothesis for this study was that calcium fortification did not affect oral $\mathrm{pH}$ changes, saliva buffering capacity, or taste perception in 10-to 14year-old children. The methodology followed in this was a controlled, blinded, non-randomised clinical trial. After calcium modification of fruit juice, there was a relatively small fall in salivary and plaque levels, as well as a substantial reduction in perceived taste by the participants. The current study's findings reported that the presence of calcium citrate Malate affected the taste of the beverage. The original drink received the highest score for taste perception. It was significant to observe, that the individuals were unable to identify differences between the two calcium-fortified subgroups, and neither of the fortified drink received an unacceptable score. It can be concluded that the alteration of beverages with calcium fortification can help against the loss of tooth enamel. The calcium salt used for this study is calcium citrate Malate, which has been authorised for food fortification by WHO guidelines. The use of calcium citrate Malate has the benefit of having a high solubility of $80 \mathrm{~mol} / \mathrm{lit} / \mathrm{lit}$. The increased solubility of calcium from calcium citrate Malate accounts for increased efficacy, both for preventive and erosive action on dental local caries ${ }^{7}$.

\section{The Salts of Calcium}

Calcium is a widely abundant mineral in the body. Bones and teeth comprise a $99.9 \%$ of calcium. Calcium deficiency can be treated using various supplementations of calcium such as calcium citrate Malate, tricalcium citrate, calcium lactate, calcium lactate gluconate, etc. The calcium are also derived from some natural resources such as coral, dolomite and oyster shell. Calcium carbonate, calcium citrate Malate, calcium chloride, calcium acetate are said to calcium equivalents. Among these $\mathrm{CaCo} 3$ and $\mathrm{CCM}$ have more elemental Ca. CCM have better absorption rate when consumed orally compared to calcium carbonate. It is recommended for those individuals with inflammatory bowel disease or absorption disorders ${ }^{8}$.

\section{Macroelements for oral health}

This chapter attempts to review the importance of these dietary macro elements on oral health and in particular their role in tooth loss, dental caries, erosive tooth wear and periodontal disease. Calcium and phosphate make up the bulk of the mineralised human tissues. Adequate intake of both is therefore of crucial importance in maintaining the health, function and retention of teeth and bones. The macro elements calcium, potassium, sodium, phosphorus, and chlorine are critical to human survival. An appropriate amount of these components regularly is required to maintain a broad array of biological processes. In terms of oral health, calcium, in particular, but also phosphate, play a vital role in the maintenance and function of the teeth. Insufficient calcium intake has also been linked to a worsening of periodontal disease severity. The aspects of sodium, potassium, and, in specific, chloride in retaining oral health, on the other 
hand, have received little attention and appear to be of minor relevance 9 .

\subsection{Calcium Citrate Malate in Immunology 10-17}

\begin{tabular}{|c|l|c|}
\begin{tabular}{c|l} 
Sr. \\
No.
\end{tabular} & \multicolumn{1}{|c|}{ Title of Paper } & $\begin{array}{c}\text { Year } \\
\text { Publis } \\
\text { hed }\end{array}$ \\
\hline 1 & Immunometabolic Circuits in Trained Immunity & 2016 \\
\hline 2 & $\begin{array}{l}\text { Mitochondrial Control of Immunity: Beyond } \\
\text { ATP. }\end{array}$ & 2017 \\
\hline 3 & $\begin{array}{l}\text { A Role for the Krebs Cycle Intermediate Citrate } \\
\text { in Metabolic Reprogramming in Innate } \\
\text { Immunity and Inflammation }\end{array}$ & 2018 \\
\hline 4 & $\begin{array}{l}\text { Metabolic Therapies Inhibit Tumor Growth in } \\
\text { vivo and in silico. }\end{array}$ & 2019 \\
\hline 5 & $\begin{array}{l}\text { NK Cell Metabolism and TGF } \beta \text { - Implications for } \\
\text { Immunotherapy. }\end{array}$ & 2019 \\
\hline 6 & $\begin{array}{l}\text { Circles of Life: Linking Metabolic and Epigenetic } \\
\text { Cycles to Immunity. }\end{array}$ & 2020 \\
\hline 7 & $\begin{array}{l}\text { Is Citrate A Critical Signal in Immunity and } \\
\text { Inflammation? }\end{array}$ & 2020 \\
\hline 8 & $\begin{array}{l}\text { The Immunologic Warburg effect: Evidence and } \\
\text { Therapeutic Opportunities in Autoimmunity. }\end{array}$ & 2020 \\
\hline
\end{tabular}

\section{Metabolic Networks in Innate Immunity Memory Responses}

Build-up of a memory response against secondary infections from related or non-related pathogens is not only mediated by $\mathrm{T}$ and $\mathrm{B}$ lymphocyte influenced adaptive immunity. This epigenetically controlled, innate immune memory response was seen in monocytes and macrophages of plants, invertebrates and mouse models. In vivo and ex vivo experiments pertaining to 'trained immunity' showed that it could ameliorate host-pathogen interactions (by enhancing cytokine production, effector functions of NK cells etc.) and strengthen innate immunity. However, inappropriate induction by endogenous triggers leads to auto inflammatory/immune diseases. The different subsets or phenotypes of $\mathrm{T}$ lymphocytes and macrophages derive energy from different metabolic pathways via synthesis of different glycolytic intermediates. Such pathways such as glycolysis, TCA cycle, glutamine, cholesterol synthesis etc are the ones trained immunity and the related therapeutic strategies are derived from. i) Glycolysis: Cancer cells goes through a metabolic switch from OxPhos to glycolysis where by its activated $T$ cells and macrophages upregulate glucose metabolism, produce lactate and elevated ATP. C. albicans product $\beta$-glucan trained monocytes induces memory innate immunity at cytokine and epigenetic levels, confirmed by using inhibitors and specific gene knockouts. $\mathrm{NAD+} / \mathrm{NADH}$ rations are enhanced here, as in the case of LPS stimulated monocytes, activating the histone deacetylases which promotes anti-inflammatory responses. But lactate end product is antagonistic, promoting euchromatinaization, promoting increased production of immunological molecules. Glycolytic enzyme
GADPH is a post-transcriptional modifier of the effectors. ii) TCA Cycle: Oxidation of various components like pyruvate occurs in the TCA cycle. It remains active upon the decrease in OxPhos rates in macrophages, continuously accumulating metabolites. One of these are citrate, which induce fatty acids and sterol synthesis, components involved in its mitochondrial to cytosolic transfer such as the transporter $\mathrm{CIC}$ and the product Acetyl-CoA function as epigenetic regulators promoting histone acetylation. Fumarate and succinate, similarly, apart from enhancing glycolysis and transcription of also act as DNA demethylase antagonists. iii) Glutamine metabolism produces intermediates like succinate, fumarate, acetyl-CoA, ATP, NADPH, Malate to influence Malate-aspartate shuttle, cholesterol and fatty acids etc. The importance of these intermediates is mostly epigenetic and up regulatory in the trained macrophages/monocytes. Similarly, aspartate metabolism induces trained immunity by influencing the epigenetics and metabolite regulation from its involvement in Malateaspartate shuttle, urea cycle, gluconeogenesis, folate and choline metabolism, purine metabolism, citrate formation from acetyl-CoA. Modification of the cell membrane integrities to increase signal transduction related to memory innate immunity can be attributed to the cholesterol metabolic pathway.

Therapeutic approaches to inhibit pro-inflammatory activities induced by the discussed metabolic pathways can be used to treat ailments such as atherosclerosis and cardiovascular disorders. They can be used to restore immunodeficiencies in the infants and elderly and can reverse haywire immune functions in diseases like post sepsis immunoparalysis. Thus, intercorrelating and studying different stages of the varied metabolic processes involved can help us exploit trained immunity to develop therapy ${ }^{10}$.

\section{Exploring the Unconventional: Mitochondrial Control in Immunology}

For many decades it was thought that a particular cell conforms itself to a status of being pro or antiinflammatory and their intrinsic metabolic systems had nothing to do but maintain their bioenergetics and perform biosynthesis of essential molecules and components. The interlinking between these two systems have been brought to light recently, creating a whole new field of" immunometabolism" who's arms have reached to not only the components of innate immunity like macrophages and dendritic cells but has also extended to the $T$ and $B$ cell components of adaptive immunity and tumour immunity. The mitochondrial signalling role pertaining to each of these broad categories have been discussed. i) Adaptive Immunity: Normally dormant T cells subsets experience a metabolic flux upon stimulation of the receptors, causing large uptake of glucose and glutamine- metabolized by glycolysis and TCA cycle. Both the CD4 T cell types enhance mitochondrial metabolism and metabolite utilization, often involving an increase in 
their dimensions to hike their proliferation. It involves of calcium influx at the TCRs forming mitochondrial ROS (mROS) in mitochondrial intermembrane space and matrix, through ETCs activating the T cells. These diffuse into the cytoplasm and cause changes in the activation of transcription factors, like HIF1 $\alpha$, NFAT and NF-kB; bringing about different inflammatory and memory functions. Metabolic control is significant for B cells to function optimally, for example antibody glycosylation requires glucose and glutamine uptake during clonal expansion or pyruvate production to support respiration and plasma cell differentiation and survival. Like the T cells, B cells also require $\mathrm{mROS}$ for activation and proliferation. ii) Innate Immunity: Both glycolysis and OxPhos is required for controlling the pro-inflammatory, anti-microbial M1 macrophages and the anti-helminthic anti-inflammatory M2 hetero type. LPS and INF- $\gamma$ induced M1s are heavily dependent on the mROS to regulate their glycolytic and ETC flux which promote their pro-inflammatory activities though their TCA events characterized by the SDH and IDH break points. M2 macrophage metabolism differs by having an unbroken TCA cycle, reduced glycolytic flu and dependency on lipid uptake, lipolysis, FAO to aid their OxPhos which is crucial for its polarization. Glutamine dependent glycolysis is also needed to facilitate polarization. Increased mROS and decreased ETC flux is also required in activation of PAMP/DAMP related inflammasomes correlated with inhibited mitophagy and autophagy and damaged mitochondria Dendritic cells and its various subsets corelate to metabolism in a similar fashion as of the macrophages, having different glycolysis and OxPhos rates for each of its tolerogenic and immunogenic subsets. Finally, the immunosuppression in a tumour microenvironment affects the mitochondrial metabolism. Tumour cells consuming the nutrients, such as glucose or glutamine, deprives the tumour infiltrating cells of substate, slowing metabolic and immunogenic functions and hence the cytotoxic effects. Restoring the nutrient availability enhances antitumour and $\mathrm{T}$ cell infiltrating efficacy.

The interconnection between immunity, metabolism and malignancy has brought about therapeutic approaches by involving mitochondrial inhibitors and gene knockouts. The inhibitor metformin has shown promise in treating LPS induced sepsis, tuberculosis and had anti-ageing implications. Similarly, other inhibitors such as 2-DG averted tumour mediated lupus and anti-PD1 enhanced tumour immunity. Tar getting the mROS and its derivatives also proves to be a promising approach toward immune therapy ${ }^{11}$.

\section{Metabolic Reprogramming in Innate Immunity and Inflammation by Glycolytic Intermediate, Citrate}

TCA cycle intermediate Citrate, after its cytosolic export from the mitochondria forms acetyl-CoA which causes dendritic cells and macrophage activation from its resting stage rapidly. These activated forms modify glycolysis and leads to the accumulation of citrate and succinate. These integral components of innate immunity also activate $T$ cells for adaptive responses. Different phenotypes/ induced stages of macrophages (M1 or M2) and dendritic cells (TLR or LPS induced) function via upregulating or downregulating their own unique metabolic pathways and producing mediators like NO and ROS for pro inflammatory immune responses. Such metabolic changes finally culminate to influencing the two major breakpoints in Krebs cycle which cause citrate accumulation ad formation of downstream reprogramming products. Finally, inhibiting the former breakpoint of SDH is the citrate product itaconate which has antibacterial and antiinflammatory properties. i) Citrate's role in inflammation: Inflammatory properties of citrate were found when its accumulation and upregulation of components such as its transporter and the ACLY enzyme was seen in immunologically stimulated macrophages. Downstream processing of oxaloacetate from citrate acted upon by ACLY produces NADPH which is needed for the production of ROS and NO, respectively. The acetyl-CoA acetylates proteins, controlling inflammatory gene expression and is converted to malonyl-CoA for Lys residue-malonylation for similar epigenetic purposes. Acetyl-CoA is also important in lipogenesis, membrane expansion necessary for DC activation and cytokine production and, the production of PGE2 in macrophages. Depletion of these above intermediates are consistent with the metabolic arrest and apoptosis of cancerous cells and macrophages while only the intermediate in one of the pathways to produced NADPH from Acetyl-CoA, IDH showed upregulation during glucose deprivation. Citrate, the subsequent fatty acid synthesis and the pentose phosphate pathway is also important for TLR and DC activation and impacts mitochondrial respiration. Citrate Malate shuttle is essential in activating Natural Killer cells. ii) Posttranslational modifications: Adding on to the ACLY mediated histone modifying roles, it noticed that the acetylation of the $\mathrm{CIC}$ transporter was enhanced in glucose deprived conditions to increase the citrate flux. Also, cytosolic product of citrate, Acetyl-CoA acetylates GADPH enzyme- increasing its catalytic activity for posttranslational modifications. All these aid in dendritic cell and macrophage activation and differentiation. iii) Itaconate: Citrate accumulation in the isocitrate dehydrogenase breakpoint of the TCA cycle is associated with the anti-inflammatory itaconate production, fatty acid metabolism and euchromatinization. Citrate is converted to cis-aconitate, who's decarboxylation produces itaconate in LPS activated macrophages with LPS with IRG1 induction. Itaconate supresses production of pro-inflammatory mediators NO, IL1 $\beta$, IL18, and HIF1 $\alpha$, which are enhanced by LPS. Its antimicrobial properties stem from its toxicity to $\mathrm{ICL}$, a key component of the glyoxylate shunt in bacteria and to inhibit SDH breaking point of Krebs cycle. Its intracellular levels are proportional to the efficacy against bacteria and therefore, a cell permeable analogue DMI is being worked with to develop pharmaceutical and therapeutic strategies. Since citrate and its downstream products and derivates prove to be a 
critical signalling substrate linking various branches of metabolism, epigenetics and immunology- there lies an ocean of networks which can be cleverly tweaked and utilized for our therapeutic requirements ${ }^{12}$.

\section{Efficacy of Antitumorigenic Metabolic Therapies in vivo and in silico}

Malignancy can be safely called a combination of a metabolic and genetic irregularity, thus leading to exploration in both the epigenetic level and the Warburg physiology. Tumour cells meeting its energy needs from the conversion of glucose to lactate gives us an opportunity to develop cancer therapies bases on metabolic reprogramming since malignant cells exhibit the features of both catabolism and anabolism- renders the achievement of complete immunosuppression difficult as there exist a plethora of interconnected metabolic networks involved. However, targeting the enzymes involved in the central carbon metabolism like pyruvate dehydrogenase (PDH) and ATP citrate lyase $(A C L)$ with preexisting drugs such as Alpha Lipoic Acid (ALA) and Hydroxycitrate (HCA), independently or in combination (METABLOC) on in synergy with RNA silencing methods and traditional chemotherapeutic drugs such as Cisplatin has shown effective results in tumour suppression in cell lines. i) Tumour volume evolution was recorded in vivo with respect to Lewis lung carcinoma cells xenograft in a mouse model with treatments beginning after 12 days of inoculation, using PBS buffer as control. A graph was plotted for tumour volume vs the time from inoculation in days. Cisplatin, slowed down tumour growth while METABLOC only showed a slight reduction. High and lowdose of Metformin slightly delay tumour growth in a dose dependent fashion. High dose Diclofenac works similarly to Metformin, even enhancing the activity of Metformin but at a low dose it shows the same results as the control. METABLOC and high-dose Metformin used in combination slows and inhibits tumour growth as well. While a combination of METABLOC, high-dose Metformin and Diclofenac significantly inhibits tumour growth and reverses the rising tumour curve. ii) Now, from the previously obtained kinetic-metabolic graph, in silico models were prepared to help us statistically predict the tumour growth rates and tumour metabolic phenotypeswhich were in fact indeed congruent with the experimental data. To bring the model in-line with metabolic therapy, artificial blood influx was also integrated to mimic the natural microenvironment of the tumours. However, such a model could not reflect volume reductions that might happen due to apoptosis. The model also predicts and showcases the metabolite concentrations upon supressing Warburg physiology with therapy. Concentrations of blood metabolites (glucose and lactate) and metabolic intermediates and products like ATP, NAD, NADP have been measured in normal and cancer cells from human colon tissues. Graphs were generated measuring the concentrations in $\mathrm{mM}$ vs the time from inoculation in days. Upon drug administration (combination of METABLOC, high-dose Metformin and
Diclofenac), blood eliminated lactic acid and then it accumulated with tumour and NAD+/NADH ratio, is the catabolic marker of aerobic glycolysis and maintains the glycolytic potential of malignant tissues, decreases upon glucose exhaustion caused by retardation glucose uptake by the therapeutic effect, followed by retardation of lactic acid secretion. The negative flux and low values of LDH corresponds to lactate uptake and the increase of the respiration flux both translate a reverse Warburg effect, thus showing a positive result of therapy.

From the dynamic in silico model showing tumour suppression and reversal of Warburg physiology on drug administration consistent with the in vivo experimentations with the same, it can be said with confidence that reducing glycolytic flux on malignant cells and manipulating the metabolic networks by designing metabolic drugs to do the same can guide us to more prospects of eliminating the metabolic irregulates that cause cancer worldwide ${ }^{13}$.

\section{Role of Anti-Inflammatory Cytokines on Natural Killer Cell Anti-Cancer Properties and}

The major component of innate immunity, the Natural Killer cells, based on its interactions with the receptors, cytokines and external stimuli provides pro-inflammatory activity against cancerous tumours. Since, just like T cells and macrophages' activation and proliferation dependencies, NK cells are associated with upregulation of metabolic processes like glycolysis and OxPhos. This draws interest to explore immunometabolic associations of cancer therapy with respect to long lost concepts and medications, since, certain checkpoint antigens influence by metabolism are expressed by NK-cells as well. Antiinflammatory cytokines such as TGF- $\beta$ may affect NK cell's metabolic reprogramming and provide an insight to exploit them for anti-cancer tumour supressing therapies. Upregulation of metabolic pathways of glycolysis and OxPhos is inculcated by native homeostatic cells when they receive an immunological trigger for activation. Similarly, dormant NK cells depend on only OxPhos but upon activation it requires both glycolysis and oxphos to meet their metabolic needs and promote longevity to work with the adaptive immune system. This includes with the major involvement of the citrate-Malate shuttle to produce $\mathrm{NAD}+$ to act as a co factor for posttranslational modifier enzyme GAPDH and NADH. NK mitochondria depolarise upon contact with a tumour cell and mitophagy, in a (ROS) dependent manner, is essential for the development of NK cell memory. NK metabolism is highly dependent on regulation by the metabolic sensor $\mathrm{mTORC} 1 / 2$ proteins. Pleotropic cytokine TGF- $\beta$ is a negative regulator of NK cells though having positive control of differentiation, healing and proliferation over other cell types. Canonical TGF- $\beta$ signalling through downstream receptor binding and activating the SMAD signalling pathway is what is controls NK cells with, but control is exerted with the little known non-canonical pathway too. Several experiments with the interplay of TGF- $\beta$ and mTORC1/ 2 show that 
chronic TGF $\beta$ treatment in humans inhibits NK cell function and metabolism with TORC1 dependency but in mice this dependency doesn't need chronic treatments. TGF- $\beta$ cells impact the antioxidative status of NK cells as in murine NK cells, ROS by TGF- $\beta$ play an essential role in the generation of NK cell anti-viral memory and promotes mitophagy. TGF $\beta$ canonical signal pathway upregulated of PGC-1 $\alpha$ expression increases in mitochondrial mass and polarisation, which is essential for NK cell IFN $p$ production. TGF- $\beta$ reduces NK cell oxphos, maximises respiration and thus, clearly promoting their anti-tumour properties. This characteristic was effective in a targeted treatment in a T cell lymphoma model. The regulatory role of TGF- $\beta$ on the calcium signalling between mitochondria and ER maintains degranulation and killing capacity of NK cells as well. Thus, upon discussion on the effects of TFG- $\beta$ with its mTORC $1 / 2$ interplay on NK cells and a major mitochondrial regulation shows a positive application of NK cells in immune and cancer therapy, graft vs host diseases etc whether by direct infusion or NK cell mediated antibody therapy. Its impact on ROS stimulation, ETC dynamic and calcium signalling opens a wide range of future diagnostic tests or cancer/genotype specific tailor-made therapies to be available in clinics ${ }^{14}$.

\section{Roles of Metabolome and Epigenome in Regulation of Immunity and Homeostasis}

Chromatin landscapes not only play a crucial role in the recognition of housekeeping functions, but the patters of euchromatinization and heterochromatinzation also influences transcriptional expression, inactivation and silencing of genes regulatory functions od metabolism and its related immunological effects. Although a vast portion of epigenetic control is unknown and still under study, the working patterns of histone modifying DNA Methyltransferases (DNMT) and the ten eleven translocations dependant on the S-adenosine methionine (SAM) and $\alpha$-ketoglutarate (aKG) metabolism bring about immunological impacts and provide prospective strategies for therapy. One-carbon metabolic processes involving folate and methionine cycles leads to formation of end products like SAM. It is important to remember that such metabolism is dependent on non-essential amino acids, which includes the highly significant serine. Serine deprivation induced cell cycle arrest in lymphocytes and tumour cells, impaired T-cell proliferation and nucleotide biosynthesis, supresses interleukin production in myeloid cells, abated inflammations and microbial attacks among many. Methionine intermediate which produces SAM is also crucial for differentiation and function of both $T$ and $B$ cells. During methyl transfer, SAM is catalysed by cytosolic or nuclear methyltransferases catalyses SAM to form the methylated substrate and S-adenosylhomocysteine (SAH), which hydrolyses back to homocysteine and adenosine. But, elevated amounts of homocysteine are antagonistic to methyltransferases activity, leading to re-methylation back to methionine. aKGDH is a multiprotein rate limiting enzyme that reacts with acetyl-CoA and NAD+ to decarboxylate aKG to form succinate through succinyl-CoA intermediate. This is promoted by increased $\mathrm{Ca}$ concentration and mitochondrial acidification. Deficiency in this process associated with the chronic aberrant inflammation of neurodegenerative disorders. Also, TLR-4 stimulation in pro-inflammatory macrophages induced the expression of aKGDH-hence, proving the consistency. Fumarate, succinate and aKG influence the superfamily of epigenetic erasers called 20G-dependent dioxygenases, which include the JMJD3 and TET proteins. aKG suppresses proinflammatory effects, promotes anti-inflammation by modulating these histone demethylases. DNA methyltransferases function by adding a methylating the $5^{\text {th }}$ carbon of the cytosine bases from SAM to make 5methylcytosines. This is followed by TET converting them into oxidized methyl cytosines. These DNMTs are also accompanied in the methylation cycles by maintenance methyltransferases, de novo methyltransferases and TET oxidases. These are abundant in both $B$ lymphocytes and $T$ lymphocytes of CD4 and CD8 hetero types. Their functions can be summarised into maintenance of DNA methylation marks during DNA replication, pharmacological or metabolic interferences; maintaining lineage stability, limiting memory and differentiation and exhaustion rates in effector CD4 /CD8 T cells; maintaining macrophage and hematopoietic stem cells proliferation and functions; prevention of auto-immune disorders- among a few. Apart from the metabolome and epigenomic control over immunity as talked about above, precise knowledge about the manipulations in the epigenetic landscapes could help bring about more insight into therapeutic applications. The ever-changing ephemeral cycles of methylation could now be looked at closely with newer single-cell technologies of mass spectrometry, next generation sequencing, etc for a better understanding of the confluence between epigenetics and immunobiology ${ }^{15}$.

\section{Investigating the Signalling Role of Citrate in Pro- inflammatory Responses and Immunomodulation}

Two major fragmentation in the Krebs Cycle has shown its importance in the regulation of immunological responses. Succinate dehydrogenase accumulating the intermediate succinate stabilises and boosts HIF1 $\alpha$ and its related ROS and helping the expression of IL1- $\beta$ genes. Secondly, accumulation of citrate at the isocitrate dehydrogenase breakpoint leads to downstream inflammatory activities. Therefore, citrate as an intermediate not only acts as a metabolic intermediate linking the carbohydrate and fat metabolic pathways but its production of cytosolic compounds such as prostaglandins, itaconate and nitric oxide leads to cytokine production, anti-inflammatory responses etc. Citrate, without much knowledge about the underlying mechanisms, have been used in the form of its salt to inhibit the growth of Gram positive and negative bacteria, zoo and phytopathogens and even a wide range of retrovirus. This property can be attributed mainly upon its effects on the lipopolysaccharide product (LPS) of Gram-negative bacteria and its impact on the macrophages with a co-relation to its calcium sequestering abilities and, on its ability to dampen replication of viral 
nucleic acids and immunological components such as a wide range of interleukins. Calcium having a directly proportional efficacy was proved when calcium citrate was shown to demolish LPS related ROS and restore antioxidative status and supressed interleukin and TNF-a production in a mouse macrophage cell line. The transporter $\mathrm{CIC}$ exchanges citrate for citrate Malate between the mitochondria and the cytosol and its encoding genes were found more in LPS enhanced macrophages than the unenhanced. CIC makes way to increase cytosolic citrate amounts which leads to production of pro-inflammation mediators like prostaglandins and cytokinin. Competitive inhibition of $\mathrm{CIC}$, even in a transcriptional level showed abatement and even reversal in non-alcoholic fatty liver disease and inflammatory steatohepatitis. Epigenetic changes caused by histone acetylation and deacetylation brought about by the formation of oxaloacetate and acyl-CoA from the cytosolic citrate pool transcriptionally activates undesirable immune modulations. Inhibition of the responsible enzyme ATP-dependent citrate lyase by suitable inhibitors such as radicicol and manipulating its acetylation, phosphorylation and Toll-like receptor involved gene regulations showcased it to be a master control in macrophage activation, differentiation, maturation and also in cell cycle progressions. Finally, posttranscriptional modifications involving citrate include histone acetylation and malonylation. These affect fatty acid and amino acid side chain modification and elongation, determine the final tertiary structure of proteins, hence their functions. Malonylation, most importantly shows its immunomodulatory abilities while boosting the GADPH on lysine residues which further leads to cytokine responses and TNF-a responses. Later part of the Krebs cycle produces the intermediate itaconate during citrate conversion to isocitrate. Its production, induced by LPS and INF- $\nu$ in macrophages, targets the breaking point of succinate dehydrogenase step, reducing ROS, RNS and even INL- $\beta$ productions by the virtue of accumulated succinate. Thus, itaconate, which might function by creating a positive or a negative feedback loop is not only an immunomodulatory driver but also has its positive Metabolic regulation by mitochondrial components like $\mathrm{CIC}$, cytosolic metabolites like Acetyl-CoA and enzyme ACLY and citrate precursor itaconate provides us an opportunity for immunomodulatory therapy. Using citrate synthase knockouts naturally impaired all the other downstream metabolic processes and Krebs Cycle also affected inflammatory responses from resident macrophages and dendritic cells. Although the area of implementation such therapies are opaque, more experimental models need to be introduced to obtain clearer view in this regard ${ }^{16}$.

\section{Derivation of Immunotherapeutic Strategies from Warburg Physiology}

The new concept of immunometabolism crated a hullabaloo in researchers when it was found out that metabolic pathways not only were mere housekeepers but provide a window to treat immunological dysregulations if cleverly engineered to respond to external stimulations. It's root lie in the "Warburg Physiology" where a stark upregulation of aerobic glycolysis was discovered in tumour cells. Similar observations extended to other immunological cells of lymphoid or myeloid origin- and more recently in proliferative stem cells and angiogenic endothelium. Here we discuss the i) Evidence and Importance ii) Mechanistic Insights iii) Inhibition and Therapeutic Strategies of glycolytic regulation of the immune system. i) Aerobic glycolysis- consistent with the "Warburg Physiology", the metabolic reprogramming has been discussed with respect to immunological cells such as the $T$ and B lymphocytes, Dendritic cells, Macrophages and the NK cells. For the T lymphocytes, the studies of the CD4 and CD8 lineages show that the metabolic upregulation is coordinated with the immune responses and a glucose deprivation negatively affect the effector functions. However, these pathways, end products and regulations vary greatly among the different subsets of the $T$ cells. Dendritic cells require the glycolytic upregulation for its survival, triggering its pro-inflammatory activities with the stimulation of its pattern recognition receptors and also to migrate from the peripheral tissues to the lymph node. M1 and $\mathrm{M} 2$ macrophages display similar Warburg physiology. With opacity in knowledge about the mature ones, naïve and quiescent B lymphocytes draw energy from OxPhos, similar to the naïve T cells. Natural Killer cells depend on both OxPhos and glycolysis, levels increasing substantially on prolonging stimulation. Though some NK cells function similar to $T$ lymphocytes in the aspect of metabolic pathway, Krebs cycle is avoided and OxPhos is supported by a citrate-Malate shuttle, who's transcriptional regulation can control fatty acid and cholesterol synthesis. From metabolic pathways providing carbon substrates to generating ATP for proliferation and anabolism, keeping in mind all the speculations and the shortcomings, a few mechanisms to link metabolism and immunology have been enlightened. These include posttranscriptional cytokine control, transcriptional control of gene networks and even direct control of glycolytic intermediates, to support inflammatory immune functions. ii) Though in clinical and pre-clinical trials, strategies to control the metabolic pathways to further control the immune responses are discussed in pharmaceutical and dietary aspects. Pharmacological techniques in animal models using glycolytic inhibitors were effective against several CD4 involved autoimmune diseases like lupus, graft rejections, psoriasis and inflammatory diseases like asthma. Immunomodulatory drug like DMGF inhibited GADPH pathway and aerobic glycolysis in human and mouse models, showing promise for clinical trials. Consistent with the results of DMF, dietary restrictions of limiting glucose showed decrease in lymphocyte counts. Methods such as ketogenic diet, Atkin's diet, intermittent fasting etc shows promising results in combating immunological irregularities. With several challenges ahead like the wide gap between in vivo and in vitro testing, human and animal models, mechanistic 
hypothesis and workbench safety issues as well as incomplete knowledge about the probable clinical drugs- it is still hopeful that a little bit of tweaking of the metabolic system can help us regulate the immunology of our bodies and fight diseases ${ }^{17}$.

\subsection{Calcium Citrate Malate in Joints ${ }^{18-26}$}

\begin{tabular}{|c|c|c|}
\hline $\begin{array}{l}\text { Sr. } \\
\text { No } \\
\text {. }\end{array}$ & Title of Paper & $\begin{array}{l}\text { Year } \\
\text { Publi } \\
\text { shed }\end{array}$ \\
\hline 1 & $\begin{array}{l}\text { Effect of calcium citrate-Malate on skeletal } \\
\text { development in young, growing rats }\end{array}$ & 1990 \\
\hline
\end{tabular}

A control trial of the effect of calcium

2 supplements on Bone density in Postmenopausal 1990 Women

Calcium Supplementation and bone mineral density in adolescent girls

Supplementation Trials with Calcium Citrate

4 Malate: Evidence in Favour of Increasing the Calcium RDA During Childhood and Adolescence

The effect of calcium supplementation and Tanner

5 stage on bone density, content and area in teenage women

Comparative absorption of calcium sources and

6 calcium citrate Malate for the prevention of osteoporosis

Effect of cholecalciferol plus calcium on falling in

7 ambulatory older men and women: a 3-year randomized controlled trial

A Randomized Open-Label Clinical Study

8 Comparing the Efficacy, Safety, and Bioavailability of Calcium Lysinate with Calcium Carbonate and Calcium Citrate Malate in Osteopenia Patients

Calsiara Tablets: A Clinical Study on Calcium Citrate Malate for Bone Health

\section{Calcium Citrate Malate's Effect on Developing Skeleton of Young Rat}

Calcium from calcium citrate-Malate (CCM), a combination of calcium, citric acid and malic acid, is preferred consumed over calcium from calcium carbonate in humans and in rodents. The current examination was intended to contrast CCM and calcium carbonate; for consequences on 56 weanling female C/D can fed on either CCM or calcium carbonate, at 0.3 or $0.6 \%$ dietary Ca for 4 or 12 weeks. There was no significant pattern for rats fed on CCM to weigh more and have bigger bones than rats fed on calcium carbonate. The $0.3 \% \mathrm{Ca}$ abstains from food (either source) brought about decreased trabecular bone volumes in tibias. After 4 wk, rats fed on CCM had 23-25\% more trabecular bone than rats fed on calcium carbonate. By 12 wk, the thing that matters was considerably more dominant i.e. rats fed on CCM had $44-47 \%$ more trabecular bone than fed on calcium carbonate. Dietary calcium source didn't influence cortical bone. It is inferred that CCM is a more bioavailable calcium source than calcium carbonate. Impacts of dietary calcium level and source on weight acquire were little in spite of the fact that there was a pattern for rats fed on CCM to weigh more than rats that fed on calcium carbonate. By 4 weeks, rats fed $0.6 \%$ Ca as CCM were fundamentally heavier than rats fed on calcium carbonate at one or the other level. After 12 weeks, the pattern for better development with CCM proceeded. Tibia and femur fat-free dry weights (FFDW) were lower, cortical bone density and region were decreased and marrow hole region was expanded in rodents fed $0.3 \% \mathrm{Ca}$ than that of $0.6 \% \mathrm{Ca}$ at 4 weeks however not at 12 weeks. In this manner, calcium and phosphorus content and conc. of tibia and femur at 4 week and in femur at 12 week were lower in rodents fed the lower calcium diet. After both 4 and $12 \mathrm{wk}$, rodent fed on CCM had altogether more trabecular bone than did rodents fed on calcium carbonate. The expanded measure of trabecular bone in the tibia metaphysis of rodents fed on CCM can be seen. Longitudinal bone development rate was fundamentally expanded in rodents fed on $0.3 \% \mathrm{Ca}$ as CCM contrasted with rodents fed on $0.6 \% \mathrm{Ca}$ as calcium carbonate at 4 week and was similar among all groups at 12 weeks. The tests revealed here give proof that CCM fabricates more trabecular bone than calcium carbonate in young, growing animals. In the event that one characterizes bioavailability as the assimilation and usage of a supplement, CCM is a more bioavailable calcium source than $\mathrm{CaCO}_{3}$. The increment in trabecular bone was seen at both peripheral $(0.3 \% \mathrm{Ca})$ and satisfactory $(0.6 \% \mathrm{Ca})$ dietary calcium levels. Since more calcium is ingested from $\mathrm{CCM}$ than from $\mathrm{CaCO} 3$ in people. CCM could repress bone resorptions a more noteworthy degree than $\mathrm{CaCO} 3$, albeit this remaining part is to be demonstrated ${ }^{18}$.

\section{A Controlled Trial to Study the Effect of Calcium Supplementation on Bone Density in Postmenopausal Women}

For this trial, a pilot study conducted, it found that healthy postmenopausal women who took less than $400 \mathrm{mg}$ calcium, lost mineral from the spine at a greater rate than women whose intake was higher. They had embraced a double-blind, placebo controlled, randomized preliminary to check the impact of calcium on bone loss from the spine, femoral neck, and radius in 301 postmenopausal ladies, half of whom had a calcium consumption lower than 400 $\mathrm{mg}$ each day and a large portion of an admission of 400 to $650 \mathrm{mg}$ each day. The ladies got placebo treatment or either calcium carbonate or CCM $(500 \mathrm{mg}$ of calcium each day) for 2 years. Dose of $500 \mathrm{mg}$ of supplemental calcium was chosen because it would bring most of the women in the lower calcium-intake group up to the level of the current RDA of $800 \mathrm{mg}$ per day. Two calcium sources were selected: calcium carbonate, because it is a widely used supplement, and CCM, because of its demonstrated bioavailability. At the dose that was tested, supplementation with CCM was more effective than supplementation with calcium carbonate. Among the latepostmenopausal ladies in the lower calcium-consumption class, the mean $( \pm S D)$ weight file was higher in the group that got calcium carbonate than that got CCM and the underlying mean $( \pm \mathrm{SE})$ bone-mineral density was greater 
in the calcium carbonate group than the CCM group at the spine and at the femoral. In ladies who had gone through menopause 5 or less years sooner, bone loss from the spine was fast and was not influenced by supplementation with calcium. Among the ones who had been postmenopausal for a very long time or more and who were given placebo treatment, bone loss was less quick in the group with the higher dietary calcium admission. In those with the lower calcium consumption, CCM prevent bone loss during the 2 years; its impact was essentially not the same as that of placebo $(P<0.05)$ at the femoral neck, radius and spine. Calcium carbonate kept up bone density at the femoral neck not spine. Healthy old postmenopausal ladies with a day-by-day calcium admission of under 400 $\mathrm{mg}$ can essentially diminish bone loss by expanding their calcium admission to $800 \mathrm{mg}$ each day. Here, supplementation with CCM was more successful than supplementation with calcium carbonate. Among these ladies, just CCM prevent bone loss from the spine, though both CCM and calcium carbonate prevent bone loss from the femoral neck and the radius. On the premise of this investigation, we suggest that sound postmenopausal ladies whose typical dietary calcium admission is low be asked to expand their calcium admission to $800 \mathrm{mg}$ each day, the current suggested dietary stipend, to restrict bone loss. At this portion, CCM was a preferable supplement of calcium over calcium carbonate for enlarging dietary calcium consumption ${ }^{19}$.

\section{Study of Calcium Supplementation and Bone Mineral Density in Adolescent Girls}

Cross- sectional studies of bone density in teenagers had suggested that rapid increases occur during puberty in both boys and girls and that peak bone density in the spine and hip of females is achieved about the age of 20 years. This aims to study the effects of calcium supplementation on bone acquisition in teenager or adolescent white girls. Initially 120 girls were selected with a mean age of $11.9+0.5$ years but later 94 girls completed the process. A randomized, double- blind, placebo-controlled trial for the effects of 18 months of calcium supplementation (CCM) on bone density and bone mass. $500 \mathrm{mg} / \mathrm{d}$ calcium as CCM was given and control received placebo pills. By use of dual energy $X$ - ray absorptiometry, total body bone mineral density (TBBMD) and total body bone mineral content (TBBMC) were measured. Similarly, bone density at lumbar spine (LSBMD), hip and wrist i.e. sites susceptible to fracture were measured. Calcium intake from dietary for the entire study group is averaged $960 \mathrm{mg} / \mathrm{d}$. The supplemented group intake, on average, an additional 354 $\mathrm{mg} / \mathrm{d}$ of calcium. The supplemented group compared with the placebo group had greater increases of lumbar spine bone density (18.7\% vs $15.8 \%$; $\mathrm{P}=.03$ ), lumbar spine bone mineral content (39.4\% vs $34.7 \%$; $\mathrm{P}-.06)$, total body bone mineral density (9.6\% vs $8.3 \%$; $\mathrm{P}=.05)$, and 24 -hour urinary calcium excretion ( 90.4 vs $72.9 \mathrm{mg} / \mathrm{d}$; P-.02), respectively. Every individual was instructed to take 2 tablets per day containing either $250 \mathrm{mg}$ of calcium per tablet or microcrystalline cellulose (placebo). $\mathrm{S}$ plus routines were used to plot growth curve models showing changes in TBBMD, TBBMC, LSBMD and LSBMC over time. By increasing the daily calcium intake from $80 \%$ to $110 \%$ via CCM resulted in noticeable increase in total body and spinal bone density in adolescent girls. This increase estimated of $24 \mathrm{~g}$ of bone gain per year during growth, which may protect against any future osteoporotic fracture ${ }^{20}$.

\section{Supplementation Trials with Calcium Citrate Malate to Study the Increase of Calcium Recommended Dietary Allowances during Adolescence and childhood}

As peak bone mass is a significant determinant of future fracture danger, the objective of the current calcium prescribed dietary stipend during youth is to give a calcium admission that permits people to arrive at their full hereditary potential for getting skeletal mass. In the most recent amendment of the suggested dietary stipends (RDA) a more prominent accentuation was put on the requirement for satisfactory calcium admission in early life (National Research Council 1989). It results from doubleblind, placebo controlled, calcium supplementation concentrates in youngsters and teenagers. Altogether cases, CCM were the supplemental source of calcium. At equimolar calcium portions, this source of calcium has been appeared to yield more prominent partial retention than milk or calcium carbonate (Miller et al. 1988 and 1989, Smith et al. 1987) and to hinder bone loss in more established postmenopausal ladies to a more prominent degree than calcium carbonate (Dawson- Hughes et al. 1990). Total calcium intake in the prepubertal CCM enhanced that of the placebo group (1615 $\hat{A} \pm 288$ versus $888 \hat{A} \pm 173 \mathrm{mg} / \mathrm{d}$ ) and was joined by more prominent mean expansions in bone density at all six skeletal destinations estimated .Conversely, expanded calcium intake was not related with essentially more prominent bone density gains in pubertal subjects despite the fact that supplementation raised all out calcium consumption from $924 \hat{A} \pm 179$ to $1593 \hat{A} \pm 185 \mathrm{mg} / \mathrm{d}$. During a half year intercession period there was no distinction between the CCM and placebo supplements subjects concerning acquire in tallness, weight or pubertal movement. Conversely, subjects who got $1000 \mathrm{mg}$ of supplemental calcium had essentially more noteworthy expansions in complete body bone mineral substance than those enhanced with just $500 \mathrm{mgCa} / \mathrm{d}$. In the intervention studies, the magnitude of increase in bone acquisition afforded by calcium supplementation above the RDA equates to approximately $10 \%$ of 1 SD in bone mass per year. From prospective studies in adults, the relative risk of fracture is known to change by as much as $100 \%$ for every 1 SD change in bone mass (Hui et al. 1989). Subsequently, the extra increase in bone related with long term calcium intake over the current RDA during youth would be anticipated to yield a critical decrease in lifetime fracture risk. Calcium absorption from CCM is known to be high (Miller et al. 1988 and 1989, Smith et al. 1987) and Dawson Hughes et al. (1990) reported that at equal molar calcium doses CCM was more effective for reducing bone loss in 
postmenopausal women than $\mathrm{CaCO}_{3}{ }^{21}$.

\section{In Teenager women: Effect of Calcium Supplementation and Tanner Stage on Bone Density, Content and Area}

112 Caucasian young ladies, $11.9+0.5$ years old at section, were randomized into 2 year, double-blind, placebocontrolled preliminary to check the impact of calcium supplementation on bone mineral content, bone zone and bone density. Supplementation was $500 \mathrm{mg}$ CCM each day. Controls got placebo pills, and consistence of the two groups found the average value of $72 \%$. Calcium consumption from dietary sources found the average value of $983 \mathrm{mg} /$ day for the whole investigation group. Supplemented group received $360 \mathrm{mg}$ calcium/day from CCM. The supplemented had more prominent increments of all out body bone measures: content $39.9 \%$ vs $35.7 \%$ (p $=0.01)$, area $24.2 \%$ vs $22.5 \%(p=0.15)$ and density $12.2 \%$ vs $10.1 \%$ ( $p=0.005)$. This investigation showed that the supplemented group had very high gain compared with the control group for bone mineral density, content and area. Specifically, in the lumbar spine and pelvis, the gain by supplemented was $12 \%-24 \%$ more prominent than the increase made by control group. Compared to the placebo group, the supplemented group had increased yearly gains of bone content, area and density which represented about $1.5 \%$ of adult female values. The percentage of girls reaching menarch didn't differ between the two study groups. After two years, (at mean age 13.8 years) $74 \%$ of the subjects had reached menarch. The placebo and calcium supplemented groups did not differ at study entry with respect to any of the variables measured. Bone acquisition was significantly greater in the calcium supplemented group than in the placebo group. The increases in lumbar spine and pelvis bone measurements of the supplemented group were $12-24 \%$ greater than those achieved by the placebo group. Acquisition of optimal peak bone mass is now regarded as possibly the most important measure to reduce the risk of future osteoporotic fractures. Fractional absorption of calcium from dairy products is about $26 \%$, whereas $38 \%$ of calcium from CCM is absorbed. Accordingly, in the present study the average bioavailable calcium per day for the placebo group would have been approximately $243 \mathrm{mg} /$ day. In contrast, the supplemented group received approximately $=270 \mathrm{mg} /$ day from dietary sources plus $137 \mathrm{mg} /$ day from CCM for a total of $414 \mathrm{mg} /$ day. This difference is notable as it represents $170 \%$ more absorbable calcium than that received by the control group. Thus, modest levels of calcium supplementation as described in this study may provide significant long-term protection against fractures in later life ${ }^{22}$.

\section{To prevent Osteoporosis a Study has done on Comparative Absorption of Calcium Sources and CCM}

Osteoporosis is a multifactorial character disease Influencing bone mass and strength. Calcium is accepted uniquely to influence bone mass, not bone strength or delicacy. Metaexaminations of calcium and bone mass investigations show supplementation of 500 to $1500 \mathrm{mg}$ calcium everyday improves bone mass in teenagers, adolescents, young adults, older men and postmenopausal women. CCM has high bioavailability and in this manner has been the subject of calcium studies in these groups. Beneficial outcomes have been seen in prepubertal young ladies, teenagers, and postmenopausal ladies. The addition of trace minerals and vitamin $D$ in isolated preliminaries has improved the impact of calcium citrate Malate on bone density and shown a decrease of crack or fracture risk. When compared with placebo, the impact of CCM in late menopausal ladies was fundamentally better compared to calcium carbonate. The CCM group had a $60 \%$ decrease in spinal bone loss while the calcium carbonate group had a $15 \%$ decrease. A tradial positions, the CCM group had a critical increase in bone mass while the calcium carbonate group had no gain. The net addition in spinal bone density in vitamin $D$ group was huge. Although the two groups had lost bone mass through out the colder time of year, the vitamin $D$ group had a overall increase in spinal bone mass of 0.85 percent $(\mathrm{Cl}$, $0.40 \%$ to $1.30 \%$. $p<0.001$ ). CCM, the most bioavailable type of calcium, has been demonstrated to be powerful in both of these territories, and more viable than calcium carbonate at slowing the rate of bone loss in post menopause. The addition of vitamin D and trace minerals to calcium supplementation is an effective way to prevent bone loss and reduce fracture risk in postmenopausal women ${ }^{23}$.

\section{A 3-year randomized trial to study the effect of cholecalciferol plus calcium on falling in ambulatory older males and females}

A meta-investigation found that cholecalciferol (nutrient D) ought to lessen falls by over $20 \%$. Little is thought about whether supplemental cholecalciferol in addition to calcium citrate Malate will bring down the drawn out hazard of falling in men, dynamic more seasoned people, and more established people with higher 25hydroxyvitamin D levels. Here, considered the impact of 3year supplementation with cholecalciferol-calcium on the danger of falling at any rate once in 199 men and 246 ladies 65 years or more established and living at home. Subjects were counted as less active physically if active physical work was beneath the middle level. Low 25hydroxyvitamin $D$ levels were delegated those under 32 $\mathrm{ng} / \mathrm{mL}$ ( $<80 \mathrm{nmol} / \mathrm{L}$ ). Long-term dietary cholecalciferolcalcium supplementation reduces the odds of falling in ambulatory older women by $46 \%$, and especially in less active women by $65 \%$. Supplementation had a neutral effect in men independent of their physical activity level. In 3 years, $55 \%$ of ladies and $45 \%$ of men revealed at any rate 1 fall. Mean +/ - SD pattern 25- hydroxyvitamin D levels were $26.6+/-12.7 \mathrm{ng} / \mathrm{mL}$ in ladies and $33.2+/-14.2$ $\mathrm{ng} / \mathrm{mL}$ in men. Cholecalciferol-calcium altogether diminished the chances of falling in ladies (chances proportion [OR], 0.54; 95\% certainty span [Cl], 0.30-0.97), yet not in men $(\mathrm{OR}, 0.93 ; 95 \% \mathrm{Cl}, 0.50-1.72)$. Fall decrease was generally articulated in less dynamic ladies (OR, 0.35; 95\% Cl, 0.15-0.81). Pattern 25-hydroxyvitamin D level didn't balance the treatment impact. During this 3-year 
local area based examination, supplementation with cholecalciferol-calcium fundamentally reduced the chances of falling by $46 \%$ among ladies, while no apparent advantage was seen in men. This sex difference in light of cholecalciferol-calcium has not been tended to explicitly in past preliminaries in light of the fact that primarily ladies were considered. On the other hand, the transient advantages of cholecalciferolcalcium on the danger of falling saw in 2 past European RCTs might be clarified by a mix of more established age, expanded delicacy, and essentially lowers pattern 25- OHD levels in their participants. All in all, cholecalciferol- calcium supplementation diminishes falls by $46 \%$ to $65 \%$ in local area abiding more seasoned ladies, yet neutrally affects falls in men ${ }^{24}$.

Clinical Study of Osteopenia Patients to Compare the Efficacy, Safety, and Bioavailability of Calcium Lysinate with Calcium Carbonate and Calcium Citrate Malate

To prevent osteoporpsis, dietary calcium supplements containing calcium citrate Malate and calcium carbonate have been suggested to patients. A calcium supplement Calcium Lysinate may have better absorption and results than other supplements. This clinical study aims to evaluate and compare the efficacy of calcium supplements in enhancing the bone mineral density (BMD) status in osteopenia patients and safety, bioavailability of calcium lysinate (CALNIFIT) with that of CCM and calcium carbonate (SHELCAL). Concentrations of Serum calcium rose gradually after administration of CCM, yet consistently arriving at a level roughly above standard by 180 min post-portion. At 225 and 270 min post- portion, increases following CCM were fundamentally more prominent like that of calcium carbonate. After administration of calcium lysinate, the time profile of serum calcium was strikingly extraordinary contrasted to the other two groups. The relative oral bioavailability of SHELCAL, F=223.15\%. Paired t-test was applied among preand post-BMD of calcium lysinate group. $P<0.0004$, which is profoundly significant ( $t=6.381 \mathrm{df}=7$ ), $P<0.0042$ for calcium carbonate bunch which is additionally genuinely critical ( $t=4.161 \mathrm{df}=7$ ), $\mathrm{P}<0.0069$ for $\mathrm{CCM}$ bunch which is likewise genuinely critical ( $t=3.776 \mathrm{df}=7$ ). Thus, there is very much improvement in the T-score of BMD of the groups. It was more critical in calcium lysinate group. In this investigation, calcium lysinate was better than both calcium carbonate and CCM in capacity to convey calcium to the circulatory system after oral organization and calcium lysinate may offer huge benefits as a dietary calcium supplement, The writing reference shows that ingestion of calcium carbonate is $22 \%$ \& that of CCM is $42 \%$, thinking about its natural calcium of $40 \%$ and $24 \%$. In instance of calcium lysinate which is having a natural calcium of $30 \%$ adding up to $250 \mathrm{mg}$ for each tablet, has shown a checked expansion in the rate of assimilation when contrasted and that of calcium carbonate. At the point when the bioavailability of calcium lysinate was contrasted and that of calcium carbonate, we found that it was $223.15 \%$, which adds up to $89.2 \%$ of calcium ingestion.
Thus, of the three formulations, calcium lysinate is showing a better improvement in BMD T-score when compared with calcium carbonate and $\mathrm{CCM}^{25}$.

\section{Calcium Citrate Malate for Bone Health: Calsiara Tablets}

CCM is most effectively consumed by the body. CCM and different supplements are fundamental for cell development and the creation and working of DNA. In light of clinical confirmations Calsiara is fundamental for Pregnancy Mother and Fetal development. This aims to evaluate the use of supplementations like vitamin D3, fructooligosaccharides, magnesium, zinc, manganese, copper, CCM fetal development in the Pregnancy. Vitamin D3 supplementation during pregnancy improves maternal nutrient D3 status and may decrease the danger of toxemia, low birth weight and preterm birth. Human milk oligosaccharides (HMOs) are bioactive glycans connected with medical advantages to both the bosom took care of baby and lactating mother. So Calsiara is suggested for fundamental supplement for pregnant mother and fetal development and also to improve mineral bone density. CCM may likewise be utilized in specific patients to ensure they are getting sufficient calcium (counting ladies who are pregnant, nursing, or postmenopausal, individuals taking certain drugs like phenytoin, phenobarbital, or prednisone). In the event that there isn't sufficient calcium in the blood, the body will take calcium from bones, in this way debilitating bones. Calcium supplementation has appeared to deliver a gainful impact in diminishing the danger of pregnancy-initiated hypertension. Calcium in enhancements may come as carbonate, citrate, lactate or gluconate, and overall has great bioavailability. Enhancements are cheap and promptly accessible. CCM is one of the key minerals you need during pregnancy alongside different nutrients and minerals, your body gives it to your child to help the advancement of crucial designs like the skeleton. Calcium citrate is utilized to prevent and to treat calcium deficiency ${ }^{26}$.

\subsection{Calcium Citrate Malate in Nephrology 27-36}

\begin{tabular}{|c|c|c|}
\hline $\begin{array}{c}\text { Sr. } \\
\text { No. }\end{array}$ & \multicolumn{1}{|c|}{ Title of paper } & \multicolumn{1}{|c|}{$\begin{array}{c}\text { Year } \\
\text { published }\end{array}$} \\
\hline 1 & $\begin{array}{l}\text { Calcium Absorption from a New Calcium } \\
\text { Delivery System (CCM) }\end{array}$ & 1987 \\
\hline 2 & $\begin{array}{l}\text { Stone-forming potential of milk or calcium- } \\
\text { fortified orange juice in idiopathic } \\
\text { hypercalciuric adults }\end{array}$ & 1992 \\
\hline 3 & $\begin{array}{l}\text { Calcium absorption from apple and orange } \\
\text { juice fortified with calcium citrate Malate } \\
\text { (CCM) }\end{array}$ & 1996 \\
\hline 4 & $\begin{array}{l}\text { The Health Benefits of Calcium Citrate } \\
\text { Malate: A Review of the Supporting Science }\end{array}$ & 2008 \\
\hline & $\begin{array}{l}\text { Assessment of Citrate Concentrations in } \\
\text { Citrus Fruit-Based Juices and Beverages: }\end{array}$ & \\
\hline 5 & $\begin{array}{l}\text { Implications for Management of } \\
\text { Hypocitraturic Nephrolithiasis }\end{array}$ & 2008 \\
\hline
\end{tabular}


Citrate, Malate and Alkali Content in

6 Commonly Consumed Diet Sodas: Implications for Nephrolithiasis Treatment

Noncitrus Alkaline Fruit: A Dietary

7 Alternative for the Treatment of Hypocitraturic Stone Formers

Effects of non-pharmacological

8 interventions on urinary citrate levels: a systematic review and meta-analysis

Dietary treatment of urinary risk factors for

9 renal stone formation. A review of CLU Working Group

10 Calcium citrate: from biochemistry and physiology to clinical applications

\section{Absorption of calcium from calcium citrate-Malate salt}

The main purpose to design this study was to determine the amount of calcium absorption from calcium citrateMalate salt against milk and calcium carbonate in humans and rats. Calcium citrate-Malate (CCM) was given to the subjects as a dry powder or as calcium-fortified juice. Results showed that in humans, calcium absorbed from calcium citrate-Malate was significantly higher as compared to calcium carbonate and milk. Furthermore, in rats calcium retention from CCM was as good as, if not better as compared to $\mathrm{CaCo} 3$ and milk. The data concluded that Calcium citrate-Malate is a great formulation to get necessary calcium either from fortified food or supplements. Total two experiments were designed on rats. The results of both instances showed statistically no significance. While, in orange juice calcium absorption was significant as compared to milk. Likewise, two experiments were conducted in women. In both experiments, calcium absorption from orange and milk was significantly higher. It had been surprisingly seen that the difference in retention values in rats was due to the difference in their age. The rats in experiment B were $30-40 \%$ younger than rats in group $A$. Differences in absorption and in the ratio of pool turnover both had a great influenced on this study. The result suggested that older animals had lower turnover than younger that had a higher turnover. To sum up, Calcium citrate-Malate (CCM)) is a good alternative of calcium. Calcium-fortified-rich-juices are clinically proved better for those individuals who can't consume dairy products on daily basis ${ }^{27}$.

The role of milk and orange juice in the formation of kidney stone in idiopathic hypercalciuric patients

Milk and calcium-fortified orange juice play a crucial part in urinary stones formation in idiopathic hypercalciuria adults. Both of these have the potential to change urine crystallization and urine electrolytes. According to findings, male and female idiopathic hypercalciuric patients were collectively well adapted to the milk and Calcium-citrate-Malate orange juice. Researchers also concluded that the ratio of urinary citrate rose in females. On the other hand, urinary $\mathrm{pH}$ concentration significantly increased in both males and females. The findings also conclude that milk and CCM-OJ which are a great source of calcium can reduce the danger of kidney stone formation in calcium-sensitive people if their use is within limits. The urine excretion on daily basis was the same for all the individuals during the treatment periods of basal, Calciumcitrate-Malate orange juice, and milk and these values were the same throughout the analysis. Women excreted a high ratio of sodium during milk consumption instead of the basal period while CCM-OJ consumption lowered the level of uric acid relative to the basal period. Results revealed that women had higher $\mathrm{pH}$ values than males throughout the study. All three groups had different salts of uric acid and the same level of Supersaturations for calcium oxalate. Calcium-fortified only increase urine $\mathrm{pH}$ and citrate excretion in hypercalciuric adults and it has no other effects on individuals as compared to milk. The level of Calcium phosphate raises due to the rise in $\mathrm{pH}$ in women consuming Calcium-citrate-Malate orange juice but this does not happen in case of milk consumption. The rise in Supersaturations is not significant in this case. Results concluded that Calcium-fortified orange juice consumption do not cause stone formation in hypercalciuric people. This means that CCM-OJ is not responsible for forming calciumcontaining kidney stones in their consumers. Coe et, al., 1992) $)^{28}$.

\section{Determine Calcium concentration from apple and calcium fortified-orange juice}

The main objective was to measure the absorbed calcium in the apple and calcium-fortified orange juice. This study was conducted on healthy women, female beagle dogs and male rats. The study showed that women adsorbed a high amount of calcium (250 mg), dogs absorbed $125 \mathrm{mg}$ of calcium, and rats absorbed $6 \mathrm{mg}$ of calcium. Results revealed that the females who consumed calcium-fortified apple juice had greater absorption than those who consumed calcium-fortified orange juice. On the other hand, calcium retention in dogs and rats was higher for CCM-Apple juice as compared to CCM orange juice. These data suggest that greater absorbability of calcium can be achieved by modifying ingredients of juices and beverages. The greater Calcium retention in rats can support rapid skeletal growth, however; this has not happened in case of dogs. It was noticed that the composition of CCM juices can be mimicked by manipulating the organic acid contents and carbohydrates composition. The Ca absorption efficiencies did not affect by organic acid profiles of CCM-AJ and CCM-OJ in the absence of carbohydrates. In contrast, the organic acid components of calcium citrate-Malate apple juice were linked with significantly higher calcium absorption. The result suggested that the test solution which mimicked calciumfortified orange juice had significantly lower calcium absorption than those which mimicked fortified apple juice. It is interesting to note that higher fructose level and lower organic acid profiles are directly linked to the greater calcium absorption efficiency. On the other hand, both apple and orange juices have the same carbohydrate content. Researcher suggests that fructose has high 
capacity to alter calcium absorption as compared to any other sugar. The conclusion of this study was limited due to the small population size. The greater amount of calcium from CCMAJ in rats and dogs motivates researchers to do further observations in human beings ${ }^{29}$.

\section{A review: bioavailability and health benefits of CCM}

This chapter contained a detailed summary of supporting science and proofs on the health benefits and bioavailability of calcium citrate-Malate. In adults, CCM slows down the risk of bone fracture and in old age it slows the risk of bone loss. CCM has been considered important for postmenopausal women. Any other calcium sources required the use of meal and supplements together in order to get health benefits. However, CCM can be used with or without food and it delivers important nutritional benefits. It controls the danger of kidney stones in kidney stone formers. The recommended consumption of calcium is different in different countries. This is just because of different foods sources, various lifestyles, traditions, environmental conditions, and different dietary intake. Calcium is compulsory for the proper functioning of every cell. It plays vital role during muscle contraction, enzyme secretion, blood coagulation, glandular secretion, vascular function, and neurotransmission. Almost $99 \%$ of calcium is present in teeth and bones in the form of hydroxyapatite Calcium. Calcium contributes in the growth and development of infants. Low consumption of calcium causes serious health issues in people of all age. Nowadays, people are using such types of foods and drinks which cannot meet their calcium needs due to which they are suffering from health issues such as pathologic bone fracture, osteopenia, etc. Both supplements and fortified juices provide the appropriate amount of calcium without adding any additional calories in the diet. According to the reported evidence, CCM plays a crucial role in bone accrual and calcium retention ${ }^{30}$.

\section{Determine Citrate Concentrations in calcium-fortified juices and beverages: Suggestions to improve Hypocitraturic Nephrolithiasis management}

Dietary intake of fortified-calcium in case of citrus juices is very valuable for hypocitraturic patients because it will enhance urinary citrate excretion. This study was designed to recommend the suitable diet to kidney stone formers by reporting citrate concentration of several beverages. Results revealed that grapefruit had maximum citrate concentration (64.7 $\mathrm{mmol} / \mathrm{L}$ ) however; Diet 7Up had the lowest citrate concentration $(7.98 \mathrm{mmol} / \mathrm{L})$. It is concluded, in patients with hypocitraturic calcium oxalate nephrolithiasis the citrus-based juices can be very effective alternative to medical administration. Furthermore, more potential studies are needed to analyze medical importance of these findings. The concentration of citrate in lemon juice was determined to be $75 \%$ lower as compared to the citrate concentration in grapefruit juice. Cranberry juice, lemon-lime Gatorade, and homemade lemonade characterized as moderate citrate beverages due to $20 \%$ to $50 \%$ citrate concentration. Orange juice (OJ), pineapple juice, reformed lemonade, and Crystal Light termed as high citrate beverages due to $80 \%$ to $89 \%$ citrate concentration. Diet Mountain Dew and Diet 7Up are termed as low citrate beverages due to below $20 \%$ of citrate concentration. The current study revealed that information on citrate concentration of commercially used beverages are inadequate while data on the concentration of citrus in several citrus juices is available. Several studies showed that citrus juices have a great impact on citrate level in urine and urinary $\mathrm{pH}$. It has been reported by many researchers that lemonade therapy and potassium citrate therapy have good effects on patients of hypocitraturic nephrolithiasis. According to a clinical point of view, citrusbased juices can be proved very effective for patents and these can replace medical treatment ${ }^{31}$.

\section{Nephrolithiasis can be cured by using soft drinks rich in} citrate, Malate and alkali content

Citrate is a well-known inhibitor for Calcium oxalate stone formation. Citraturia is highly affected by citrate and alkalirich diet. This study was designed to recommend the diet sodas (rich in citrate, Malate, alkali) to nephrolithiasis patients for the prevention of uric acid and calcium stones. The study involved total 15 diet sodas. Results revealed that various sodas contained an equal amount of citrate and alkali for the treatment of hypocitraturic calcium and uric acid nephrolithiasis. No significant correlation was found between $\mathrm{pH}$ and alkali content. Findings showed that frequently consumed diet sodas are rich in citrate and total alkali that are beneficial for patients with hypocitraturic and nephrolithiasis. Several types of diet sodas contained a much or greater citrate as alkalis such as diet 7up, sprite zero, diet Canada, diet Sunkist Orange, Sierra Mist Free, Fresca, and Diet Mountain Dew. In addition, coke zero, diet coke lime, caffeine free diet Pepsi had the lowest citrate as alkali. On the other hand, Fanta orange zero contained moderately less citrate as alkali as compared to lemonade. Potassium citrate has high content of citrate and alkali that increased citraturia level and urine $\mathrm{pH}$. Therefore, potassium citrate is crucial for the treatment of hypocitraturic nephrolithiasis and uric acid stones. Many studies reported that homemade lemonade beverage is effective for the treatment of Hypocitraturia patients because it can increase the urinary citrate level. Malate promotes citraturic levels by adding bicarbonates to the body. This study concludes that various diet sodas have high citrate as alkali, Malate as alkali, and total alkali as compared to lemonade beverages (Eisner et, al., $2010)^{32}$.

\section{Hypocitraturic Stone Formers can be controlled by Noncitrus Alkaline Fruit (A Dietary Alternative)}

Different fruits and vegetables are rich source of citrate, Malate, potassium, fiber, vitamin and bicarbonate. This study was specially designed to compare the special effects of noncitrus fruit and citric fruits on citraturia and some other parameters. Total 30 hypocitraturic patients were used to collect their urine samples. Results revealed that urinary citrate was significantly increased in all groups 
however, a significant increase of gastrointestinal alkali absorption, urinary potassium, $\mathrm{pH}$ were observed only in case of lemon and melon juice consumption. According to the findings, urinary citrate excretion is higher in case of melon juice and this is also similar in case of orange which is the best alternative to melon for the treatment of hypocitraturic patients. To begin with, melon juice had higher level of $\mathrm{pH}$ than orange juice. On the other hand, lime juice had more citric acid content than melon and orange juice. Findings revealed that there was no significant difference observed in the mean area under the curve (AUC) for the melon, orange and lime. It is interesting to note that orange had a significantly higher AUC for urinary sodium. The loss of Potassium has been directly linked with the loss of citrate in Hypocitraturic Stone Formers. It has been reported by various researchers that the noncitrus fruits are a great source to control calciumstone formation. Fresh lime juice has been considered more advantageous than orange juice because it is low in calories. It has been observed that orange has significantly higher sodium excretion as compared to lime. It has been recommended that lime is a good alternative for the treatment of Hypocitraturic due to its high citric acid content. Melon is also a good dietary alternative to orange due to increased urinary citrate excretion for calcium phosphate stone-formers (Leandro et, al., 2012) ${ }^{33}$.

\section{A review of CLU working group: Management of risk factors responsible for urinary stone formation}

The main objective of this review was to measure the benefits of calcium rich diet for kidney stone formers to prevent the urinary stone diseases. A systematic search of the PubMed literature was conducted on dietary treatments of urinary risk factors in order to lower down the effects of calcium oxalate stones. Results revealed that the evidences from the selected studies are not sufficient to draw a conclusion. Therefore, authors developed an additional statement as an expert opinion. It is concluded that all the patients with nephrolithiasis should be under proper evaluation about the level of calcium. It is suggested that more than $1 \mathrm{~g}$ calcium per day should be added in the diet of patients with kidney stone. Moderate dietary salt restriction is very important to control the overall urinary calcium excretion therefore; it plays a vital role to avoid calcium oxalate stone. Calciuria may also be decreased by intake of low-normal protein. Omega-3-fatty acid also plays an important role to decrease Calciuria. It has been noticed that a diet low in oxalate can decrease the urinary oxalate elimination or a diet rich in oxalate can increase the excretion of urinary oxalate. On the other hand, vegetarian diet can enhance urinary oxalate excretion. However, excretion of urinary oxalate can be decrease by lowering the intake of protein. It has been reported that after restriction of dietary protein and purine, the excretion of uric acid is decreased in the patients with renal calcium stone. In case of hypercitraturia, the administration of alkaline-citrates salt should be necessary for the medical treatment of kidney stone formers. Increased intake of fruits and vegetables increase citrate excretion and involve protection against the danger of stone formation. To conclude, proper diet can mitigate the danger of kidney stone and lower the medical treatment ${ }^{34}$.

\section{A systematic review and meta-analysis: Urinary chemistries are highly affected by non-pharmacological interventions}

The purpose of this systematic review and meta-analysis is to quantify and summarize the data on the outcomes of non-pharmacological interventions (NPIs) on urinary citrate and nephrolithiasis. Soft drinks, a high-fiber food, fruit juices, low-protein food, plant extract, and mineralrich water were used in this meta-analysis study. Among all these interventions, commercial fruit juices had high citraturia levels and show high heterogeneity. On the other hand, other interventions didn't show high $I^{2}$. Results concluded that more trials are needed to analyze the consequences of NPIs on urinary citrate levels and nephrolithiasis. Results revealed that fresh fruit drinks and other commercial drinks have increased urinary citrate levels in hypocitraturic stone formers. In addition, mineral water and a high-fiber food had non-significant changes in Urine citrate levels. In case of a low-protein diet, there was no increase in urinary chemistries. However, plant extract showed a small effect on citraturia levels. It is interesting to note that high heterogeneity and an increase in Urine citrate levels showed by Commercial fruit juice interventions which was not expressed in any other intervention. The authors were still finding shreds of evidence to support the non-pharmacological interventions in order to increase urinary citrate levels and measure nephrolithiasis risk factors. It has been cleared from the findings that the effects of non-pharmacological interventions on urinary chemistries in both healthy and stone formers were still undefined. Lemon juice is very important to prevent the formation of nephrolithiasis and hypocitraturic. Our review suggests the need for methodological improvements due to insufficient information and pre-invention data regarding the citrate content ${ }^{35}$.

\section{Biochemistry, physiology and clinical application of calcium citrate}

Dietary sources are a great source to achieve appropriate calcium that is compulsory for the body. People who have week bones and are at risk of fractures usually consume low calcium diet. Nowadays, dietary products that contain calcium citrate and calcium carbonate complexes are more common than various types of calcium salts. Even though, there are very little evidence available on the long-term safety of calcium citrate-Malate, these can be proved essential to manage several chronic pathological conditions. The main aim of this review was to identify the clinical applications of calcium citrate. This study mainly focused on the calcium citrate supplementation in osteoporosis, achloridria, hypocitraturic and bariatric patients. Calcium supplements become necessary for those individuals who do not intake proper calcium. 
Calcium supplements are absorbed through the intestine. The solubility of salts is reliant on $\mathrm{pH}$ values. By increasing the level of $\mathrm{pH}$, the solubility of both calcium decreases. The digestion of calcium citrate is greater than the calcium carbonate absorption. It has been reported that the high dose of supplements can cause serious health issues such as: constipation and flatulence, renal diseases, and cardiovascular diseases. So, the use of supplements should be within limit. Citrate plays crucial role in oxidative metabolism and is an important substrate for the energy metabolism. Citrate also involves in urinary crystallization process. Citrate has been considered vital component for healthy bones. It acts as an inhibitor in kidney stone diseases. Actually, it has been measured by some authors that low bone mass is directly linked with urinary citrate excretion. It has been analyzed through this review that calcium citrate lacks data about the risk of fragility fractures. It is no clinically proved that calcium citrate can improve clinical management of individuals diseased by hypoparathyroidism as compared to calcium carbonate. It is conducted that calcium should be the primary supplementation for the hypochlroridric patients in order to enhance their potential efficacy. Finally, Calcium citrate should be used as calcium integration for all ages of individuals.

\section{CONCLUSION}

This research review's purpose is to help the reader understand different aspects posed by the research on the Clinical Applications and properties of Calcium Citrate Malate. This is significant because it gives insights about the clinical applications of Calcium Citrate Malate in Joints, Nephrology, Dental Care and Immunology. There has been much research and discussion conducted on these opinions, pertaining to the different properties of CCM that make it so clinically valuable. Most of the research found was on the positive effects of supplementation of Calcium Citrate Malate on Osteoporosis in adults and children, prevention of renal stone formation and the role of CCM in inflammation and immunology. More research and testing are required to gain a better understanding of the Clinical Applications and properties of Calcium Citrate Malate.

Acknowledgement: We would like to thank our supervisor/guide Bharat Kwatra, from Invenzion Labs Inc. whose expertise was invaluable in formulating the research questions, methodology and drawing conclusions. His insightful feedback and guidance pushed us to sharpen our thinking and brought our work to a higher level.

\section{Human and Animal Rights}

No Animals/Humans were used for studies that are base of this research.

\section{Availability of Data and Materials}

The author confirms that the data supporting the findings of this research are available within the article.

\section{Funding Acknowledgement and Conflict of Interest}

The authors whose names are listed immediately above certify that they have NO affiliations with or involvement in any organization or entity with any financial interest (such as honoraria; educational grants; participation in speakers' bureaus; membership, employment, consultancies, stock ownership, or other equity interest; and expert testimony or patent-licensing arrangements), or non-financial interest (such as personal or professional relationships, affiliations, knowledge or beliefs) in the subject matter or materials discussed in this manuscript.

\section{REFERENCES}

1. Litonjua LA, Andreana D, Bush PJ, Cohen RE. Restorative Dentistry Tooth wear: Attrition, erosion, and abrasion.

2. Krall EA, Garcia RI, Dawson-Hughes B. Increased risk of tooth loss is related to bone loss at the whole body, hip, and spine. Calcif Tissue Int. 1996 Dec;59(6).

3. Grenby TH. Lessening dental erosive. Vol. 104, EUROPEAN JOURNAL OF. 1996.

4. Rugg-Gunn AJ, Maguire A, Gordon PH, McCabe JF, Stephenson G. Comparison of Erosion of Dental Enamel by Four Drinks Using an Intra-Oral Applicance. Caries Res. 1998;32(5).

5. Krall EA, Wehler C, Garcia RI, Harris SS, Dawson-Hughes B. Calcium and vitamin D supplements reduce tooth loss in the elderly. Am J Med. 2001 Oct;111(6).

6. Reinwald S, Weaver CM, Kester JJ. The Health Benefits of Calcium Citrate Malate: A Review of the Supporting Science. In 2008.

7. Franklin S, Masih S, Thomas AM. Effect on oral pH changes and taste perception in 10-14-year-old children, after calcium fortification of a fruit juice. Eur Arch Paediatr Dent. 2015 Dec 29;16(6)

8. Trailokya A, Srivastava A, Bhole M, Zalte N. Calcium and Calcium Salts. Vol. 65, Journal of The Association of Physicians of India घ. 2017.

9. Lippert F. Chapter 3: Macroelements: $\mathrm{Ca}, \mathrm{Na}, \mathrm{K}, \mathrm{P}, \mathrm{Cl}$. In 2020.

10. Arts RJW, Joosten LAB, Netea MG. Immunometabolic circuits in trained immunity. Semin Immunol. 2016;6.

11. Mehta MM, Weinberg SE, Chandel NS. Mitochondrial control of immunity: beyond ATP. Nat Rev Immunol. 2017;17.

12. Williams NC, Neill LAJO, Munder M, Gutenberg-universität J. A Role for the Krebs Cycle Intermediate Citrate in Metabolic Reprogramming in Innate Immunity and Inflammation. Front Immunol. 2018;9(February):1-11.

13. Moreira V, Hamraz M, Abolhassani M. Metabolic therapies inhibit tumor growth in vivo and in silico. Nature- Sci Reports. 2019;(July 2018):1-10.

14. Slattery K, Gardiner CM. NK cell metabolism and TGFß implications for immunotherapy. Front Immunol. 2019;

15. Huang SC. Circles of Life : linking metabolic and epigenetic cycles to immunity. Immunology. 2020; 
16. Zotta A, Zaslona Z, Neill LAO. Is Citrate A Critical Signal in Immunity and Inflammation ? J Cell Signal. 2020;1(3):87-96.

17. Kornberg MD. The immunologic Warburg effect : Evidence and therapeutic opportunities in autoimmunity. Wiley Syst Biol Med. 2020;(February):1-17.

18. Kochanowski BA. Minerals and Trace Elements Effect of Calcium CÃ ${ }^{\oplus}$ trate-Malateon Skeletal Development Âin Young, Growing Rats1 [Internet]. 1990. Available from: https://academic.oup.com/jn/article/120/8/876/4754540

19. Dawson-Hughes B, Dallal GE, Krall EA, Sadowski L, Sahyoun $\mathrm{N}$, Tannenbaum S. A Controlled Trial of the Effect of Calcium Supplementation on Bone Density in Postmenopausal Women. N Engl J Med. 1990 Sep 27;323(13).

20. Lloyd T. Calcium Supplementation and Bone Mineral Density in Adolescent Girls. JAMA J Am Med Assoc. 1993 Aug 18;270(7).

21. Ahdon MB, Lloyd T, Matkovic1 V. Symposium: Required versus Optimal Nutrient Intakes: A Look at Calcium Supplementation Trials with Calcium Citrate Malate: Evidence in Favor of Increasing the Calcium RDA During Childhood and Adolescence1'2 [Internet]. Vol. 124, J. Nutr. 1994. Available from: https://academic.oup.com/jn/article/124/suppl_8/1412S/4 730465

22. Lloyd T, Martel JK, Rollings N, Kulin H, Demers LM, Eggli DF, et al. Osteonorosis International The Effect of Calcium Supplementation and Tanner Stage on Bone Density, Content and Area in Teenage Women. Vol. 6. 1996.

23. Patrick L, -Associate Editor N. Permission Comparative Absorption of Calcium Sources and Calcium Citrate Malate for the Prevention of Osteoporosis. Vol. 4, Alternative Medicine Review $\bullet 1999$.

24. Bischoff-Ferrari HA, Orav EJ, Dawson-Hughes B. Effect of Cholecalciferol Plus Calcium on Falling in Ambulatory Older Men and Women. Arch Intern Med. 2006 Feb 27;166(4).

25. Shankar K, M S, Raizada P, Jain R. A Randomized Open-Label Clinical Study Comparing the Efficacy, Safety, and Bioavailability of Calcium Lysinate with Calcium Carbonate and Calcium Citrate Malate in Osteopenia Patients. J Orthop case reports. 2018;8(4):15-9.

26. Sampoorna DM, Bhavani S V., Jayasurya K, kumar BK. Calsiara Tablets: A Clinical Study on Calcium Citrate malate for Bone Health. Asian J Appl Sci Technol. 2020;04(04):12735.
27. Smith KT, Heaney RE, Flora L, Hinders SM, Procter the. Calcium Absorption from a New Calcium Delivery System (CCM). Vol. 41, Calcif Tissue Int. 1987.

28. Coe FL, Parks JH, Webb DR. Stone-forming potential of milk or calcium-fortified orange juice in idiopathic hypercalciuric adults. Vol. 41, Kidney International. 1992.

29. Andon MB, Peacock M, Kanerva RL, De Castro JAS. Calcium absorption from apple and orange juice fortified with calcium citrate malate (CCM). J Am Coll Nutr. 1996 Jun 1;15(3):313-6.

30. Reinwald S, Weaver CM, Kester JJ. The Health Benefits of Calcium Citrate Malate: A Review of the Supporting Science. In: Advances in Food and Nutrition Research. Academic Press Inc.; 2008. p. 219-346.

31. Haleblian GE, Leitao VA, Pierre SA, Robinson MR, Albala DM, Ribeiro AA, et al. Assessment of citrate concentrations in citrus fruit-based juices and beverages: Implications for management of hypocitraturic nephrolithiasis. J Endourol. 2008 Jun 1;22(6):1359-66.

32. Eisner BH, Asplin JR, Goldfarb DS, Ahmad A, Stoller ML. Citrate, Malate and Alkali Content in Commonly Consumed Diet Sodas: Implications for Nephrolithiasis Treatment. J Urol. 2010 Jun;183(6):2419-23.

33. Baia LDC, Baxmann AC, Moreira SR, Holmes RP, Heilberg IP. Noncitrus alkaline fruit: A dietary alternative for the treatment of hypocitraturic stone formers. J Endourol. 2012 Sep 1;26(9):1221-6.

34. Prezioso D, Strazzullo P, Lotti T, Bianchi G, Borghi L, Caione $P$, et al. Dietary treatment of urinary risk factors for renal stone formation. A review of CLU Working Group. Vol. 87, Archivio Italiano di Urologia e Andrologia. Edizioni Scripta Manent s.n.c.; 2015. p. 105-20.

35. Pachaly MA, Baena CP, Buiar AC, De Fraga FS, Carvalho M. Effects of non-pharmacological interventions on urinary citrate levels: A systematic review and meta-analysis. Vol. 31, Nephrology Dialysis Transplantation. Oxford University Press; 2016. p. 1203-11.

36. Palermo A, Naciu AM, Tabacco G, Manfrini S, Trimboli P, Vescini $F$, et al. Calcium citrate: from biochemistry and physiology to clinical applications. Vol. 20, Reviews in Endocrine and Metabolic Disorders. Springer New York LLC; 2019. p. 353-64.

Source of Support: The author(s) received no financial support for the research, authorship, and/or publication of this article.

Conflict of Interest: The author(s) declared no potential conflicts of interest with respect to the research, authorship, and/or publication of this article. 